\title{
ON CEREBRAL MOTOR CONTROL: THE RECOVERY FROM EXPERIMENTALLY PRODUCED HEMIPLEGIA ${ }^{2}$
}

\author{
ROBERT OGDEN AND SHEPHERD IVORY FRANZ
}

From the Physiological Laboratory of the George Washington University, and the Government Hospital for the Insane

Attention has recently been directed to the possibility of recovery of voluntary muscular control in human cases of cerebral hemiplegia. ${ }^{2}$ The results which have been reported are so different from those which have been predicted by neurologists that the whole matter of cerebral control again comes to the fore as a problem of intense practical as well as theoretical interest. It has long been believed that if improvement in motor ability does not occur in man within a period of two years following the cerebral accident the paralysis is permanent. The series of cases which have been reported show that this is not true, because even in cases of paralysis of eight or more years' duration, considerable improvement follows suitable remedial measures of the nature of exercise, including massage.

It is well known that an animal which has had its so-called motor cortex destroyed or the pyramidal fibers cut on one side shows a condition similar to that of the human apoplectic hemiplegia. It is also known that, even though the hemiplegia be complete, recovery of (voluntary) motor function takes place. The beginning of this recovery in the dog comes in a day or two, and after a few weeks the dog can use the legs on the paralyzed side apparently as well as those on the non-paralyzed side. The animal does, however, use the legs of the non-paralyzed side

1 This investigation was made possible by a grant to one of us (F.) by the Carnegie Institution of Washington, and we beg to express our sense of obligation for the assistance thus given.

${ }^{2}$ S. I. Franz, M. E. Scheetz, and A. A. Wilson: The possibility of recovery of motor function in long-standing hemiplegia. Jour. of Amer. Med. Assn., 1915, vol. $65,2150-2154$. 
in preference to those of the paralyzed side even though the latter be "recovered." And also, when the animal is under the influence of certain toxic agents, such as alcohol or ether, the previously paralyzed limbs exhibit motor disturbances even though prior to the administration of the alcohol or ether the animal appeared to be perfectly normal in a motor way. The recovery in the monkey and ape is less rapid than in the dog, although after nine to twelve months it may not be possible on casual inspection to notice any motor disturbances. ${ }^{3}$

The effect of suitable exercises in the long-standing human hemiplegics suggested that if the paralyzed segments of an animal with an experimentally produced hemiplegia were adequately dealt with the recovery would be more rapid and more complete than if the animal were permitted to recover by itself. The suggestion was tested and the results of the observations are given in the subsequent paragraphs.

Four male monkeys (macacus rhesus) about a year and a half old were successfully used as subjects. One other animal died too soon after the operation to make the results of value. The hemiplegia was produced under ether anesthesia by the destruction of the motor cortex with an electric thermocautery. The extent of the motor area was determined by faridization (bipolar), and the area destroyed corresponded with the electrical delimitation of the motor zone. After the destruction different parts of the destroyed area, and beyond, were stimulated to see if the electrical stimulations would then produce movements, and in one case in which movements were obtained the area of destruction was extended and the part already cauterized was gone over again with the cautery. To destroy the motor zone lying concealed within the central fissure the white hot cautery was pushed about 6 to $8 \mathrm{~mm}$. into the brain substance and carried close to and parallel with the fissure.

The lesions which were produced were different from those produced by cerebral hemorrhage in man (apoplexy) in that they

${ }^{3}$ For a general account of the phenomena of recovery from cerebral paralysis in the dog and monkey see Luciani: Human Physiology (Trans. by F. A. Welby), vol. 3, pp. 581 ff. (dog) and pp. 586 ff. (monkey). 
were cortical, and entirely so as far as this could be done. It will be remembered that most of the hemiplegia-producing cerebral hemorrhages in man are in the lenticulo-striate region, the cerebral insult producing in those cases interferences with or destructions of the pyramidal fibers. The lesions also differed from many in man in that in man a hemorrhage may act by pressure to produce a temporary alteration in conductivity or irritability which simulates the effect of destruction. This alteration in conductivity or irritability may partly or wholly disappear when the clot becomes organized or is absorbed. Since there is no regeneration of the cellular elements the experimental destruction may be regarded as the more complete and the more satisfactory.

Information regarding the methods of dealing with the animals are given in the brief accounts of the observations which follow. The post-operative management of the cases differed in order that the value of different procedures might be determined. Each animal was operated upon under a general anesthetic, and the later feeding and care was the same for all, except in those cases when it became necessary to feed the animal by hand.

\section{Experiment 1}

Monkey 1. Before operation this animal appeared to be righthanded, although the observations were not sufficient in number to make this perfectly certain. It was normal and lively. The left motor cortex was cauterized May 30, 1916. The animal was then obviously hemiplegic on the right side, the paralysis extending to the face as well as to the arm and leg segments. The right arm and leg were flaccid and the right side of the face drooped. The animal could not feed itself with the right hand and arm.

Management and results. The left (normal) arm was strapped to the trunk by means of a jacket so constructed that the arm could not be used for any of the important operations of feeding and climbing. The left leg could not be hampered in the same manner although it was thought to be desirable. The object of the restriction was to compel, if possible, the animal's use of the corresponding paralyzed segment. In addition to this passive method of treatment, efforts were made to get 
the animal to move the paralyzed segments. The flaccid arm, for example, was dealt with in the following manner. The animal was held by a strap attached about the waist and the dorsal surface of the right hand was struck with a strap; this appeared to "anger" the animal and he endeavored to escape from the irritation (by the use of shoulder and arm muscles), and to lift the arm and hand to grasp the irritating stimulus; subsequent to the attempts to get the animal to move the arm muscles, the muscles which in human hemiplegic cases are those most difficult to recover (the extensors) and the nerves of those muscles were stimulated by friction and tapotement from five to ten minutes, the duration of the treatment depending upon the conditions of the involved muscles. The treatment of the leg was as follows: The animal was strapped to a table, the right leg was held and the sole of the foot was struck to cause the animal to withdraw it; friction was also applied to the nerves and to the muscles; reactions similar to those of the arm segment were obtained from the leg, the animal attempting to escape from the stimulus by drawing up and by abducting the leg. At the same time the animal was led around by its strap, and in this way the animal was encouraged to use its paralyzed leg in walking and its paralyzed arm for support as it went about the room.

At first little or no reaction was obtained from the stimulating treatment, but soon the application of the stimulus brought about slight appropriate or adequate responses, and after a few days the responses to the stimuli were almost equal to those of a normal animal. Soon also the animal began to use the arm for grasping food and in carrying it to the mouth, and the arm and the leg were used but of course awkwardly at first, for climbing and holding.

At the end of fourteen days the animal could use its leg and arm very well, and three weeks after the operation the monkey was able to pick small objects from the floor and to convey them to his mouth; he was able to use the two legs, both individually and together, very well, and there was no observable disturbances in walking and climbing beyond that to be expected from an animal which had one arm (the left normal one) rendered useless by the restricting jacket. In three weeks the monkey's movements on the right side were as accurate, precise, and forceful as those of a normal animal, and when the left strapped-down arm was liberated it was found to be less accurate than the right (disuse phenomenon). About two months later this animal was observed to cateh with the right hand a fly that had alighted 
in the monkey's cage. The coordination and quickness for the performance of this act will readily be appreciated.

Summary. By preventing movement of the normal arm and then "compelling" the animal to move the paralyzed segments, and by mechanical stimulation of the peripheral nerves and of the muscles, in three weeks the animal recovered from its paralyzed condition to such an extent that the movements on the paralyzed side were judged to be normal.

\section{Experiment 2}

Monkey 1. A week after this animal had thoroughly recovered the use of its right side a second similar operation was performed on the right hemisphere. The whole of the right stimulable cortex was destroyed under asepsis and general anesthesia, June 26, 1916. The paralysis involved the left side of the face as well as the arm and leg, and the paralysis was typical of the upper neuron type.

Management and results. The right (recovered from paralysis) side of the body was not restrained and the left half of the body was not given any special treatment. In this respect the animal was given the chance to recover by itself without interference. The animal usually lived in a cage (90 by $58 \mathrm{~cm}$., and $114 \mathrm{~cm}$. high) by itself so that it would not get the stimulus of combat, etc., with another animal, but it was let out into a large room for exercise each day for periods varying from one to four hours. Some forced exercise of the newly paralyzed parts could not be prevented, for it was necessary to compel the animal to come close for observation and for testing, and its solitary living had made it somewhat timorous although during the period of its former paralysis it had been handled with relative ease. The animal, therefore, cannot be said to have been entirely without some of the treatment which had been given to it following its first hemiplegia, although this kind of treatment was given as little as possible.

Even though the animal had received a small amount of forced exercise it has remained paralyzed and apparently without much capability of using its left arm and hand (December 24, 1916). It can walk and jump; it climbs on the wire netting of its cage, it uses the left arm for a prop, and with the left hand takes hold of its strap when the latter is pulled upon. It tends to fall towards the left side, when it jumps it does not always reach the cage or box which it apparently attempts to reach, when it climbs over its cage the right arm and hand 
are used for pulling and the left is apparently used only for support. When food is given, even though the food be close to the left hand, the animal always reaches for the food with the right. Unlike a normal monkey which grasps and holds food with both hands and feet, this animal uses only the right hand and the right foot. When compelled to stand the animal holds the left arm limp at its side, the right grasps the strap to support itself. When standing the toes of the left foot are spread, the great toe is at times doubled under the foot, and the leg is used uncertainly. When excited, as by some special stimulation or when a stick is pointed at him, the monkey will jump away and in the excited condition the left arm and leg appear to be used to much better advantage than in the unexcited condition. This may be due to the predominance of reflex activity at these times. If swung from his strap above the floor he also attempts to grasp the strap with his left hand, but only a slight amount of force is necessary to disengage that hand, although the right hand holds very firmly and cannot be easily removed from the strap.

Summary. This experiment with a hemiplegic animal without special management and treatment shows that the animal may remain for a period of six months or more without very much improvement in voluntary control. This is in direct opposition to the results obtained with the right side of the same animal which, under treatment, recovered in three weeks.

\section{Experiment 3}

Monkey 2. The left motor cortex was destroyed June 2, 1916, the operation being similar to those of the first two experiments. The animal then exhibited an upper neuron paralysis, involving the face and the upper and lower extremities. The right arm was at first completely useless, the right leg was limp. In coming out from the effects of the anesthetic the animal immediately used the left arm.

Management and results. The unparalyzed side of the animal was not restrained, and in this respect the experiment was the same as in experiment 2. The animal did, however, receive "general" massage of the affected limbs, the parts being rubbed daily and the muscles being carefully kneeded. No special effort was made to get the animal to use the paralyzed segments, and the stimulation exercises like those in experiment 1 with monkey 1 were not carried out. The treatment (general, instead of special types of, exercises and massage) was carried out regularly for twenty-six days, and at the end of that time the 
monkey used the left hand exclusively for all operations. The right hand showed marked wrist drop, there was very little strength in either flexion or extension of the fingers, and the whole arm segment had not advanced much towards recovery during the period of the treatment. The leg showed a similar condition. There was a dragging of the foot when the animal crawled or attempted to walk over the floor, and the foot and leg could not be used with any facility for climbing or other kinds of operations which a normal monkey performs. It was evident, however, that some improvement was taking place, and that there would be a recovery in time seemed to be a justifiable conclusion.

Summary. General massage for twenty-six days of the paralyzed segments of an hemiplegic monkey did not bring about a recovery of motor ability, although there was some evidence of returning function, much more than that found in monkey 1 after six months' "laissez faire" treatment.

\section{Experiment 4}

Monkey 2. At the time of the second operation on this animal it was in the condition just described. The second operation was the cauterization of the right motor cortex on June 28, 1916. This resulted in a complete paralysis of the left side with characteristic flaccid condition of the arm, face and leg.

Management and results. The right (not completely recovered paralyzed) arm was bound closely to the body and only the left arm could be used by the animal for the purpose of feeding and climbing about its cage. In addition, active movements of the extensor muscles were invoked by mechanical stimulation, and massage was used for the muscle groups and for the nerves. The conditions of treatment were the same as in the first experiment with monkey 1.

The results of this treatment for twenty-six days were evidenced by great activity on the part of the animal, by its ability to use the newly paralyzed segments, and the movements could not be said to be different from those of a normal animal. The movements are accurate and of good force, and the animal dominated monkey 3 which had been in the same cage with him for some time. He now uses his legs very well in walking, he jumps more accurately than the other two animals which still survive, and he is very much more active. There is an apparent preference for the use of the left hand in feeding, but when food is withheld until the animal uses the right hand for grasping it, it is seen that the right is used apparently equally well. At the 
present writing the animal appears in all respects to be normal, there having been a continued betterment of the right side since the special exercises on that side were stopped.

Summary. This animal with hemiplegia was given special exercises with massage and it was compelled to use the paralyzed segments; voluntary ability to move the paralyzed segments returned in twentysix days, the recovery being present and apparently permanent five months after the operation.

\section{Experiment 5}

MLonkey 3. On June 2, 1916, the left cerebral motor cortex was gauterized as completely as possible. The paralysis was the same as in the previous experiments in that there was an evident complete hemiplegia of the upper neuron type of the whole right side.

Management and results. The left arm was strapped to the body of the animal so that movements of the paralyzed right side would be necessary for feeding and climbing. No other kind of treatment was given, the animal being permitted to recover "spontaneously." After twenty-six days the amount of recovery was slight. Some movement of the paralyzed arm and leg was possible, but the animal was obviously incompetent on the right side. There was a characteristic wrist drop and there was some atrophy and an extreme weakness of the right arm. The leg was moved more than the arm, but it also was weak and the movements were uncertain and rather gross in nature. This was the condition on June 28 , after which time active treatment of the right arm and leg was instituted, the treatment consisting in darly muscle and nerve stimulation by vibratory digital means, and in the stimulation of the animal by the special method already described. This treatment was continued for four weeks and in that period of time all evidence of the paralysis had disappeared, and the leg and arm had regained their normal power and precision.

Summary. The normal arm of a paralyzed monkey was restrained but no special treatment of the paralyzed segments was given for a period of about four weeks, and this management did not bring about a return of motor function. During the next four weeks the nerves and muscles were stimulated and the animal was encouraged by special stimulations to use the arm and leg. During the second month the treatment brought about a complete return of motor function so that the animal's movements became normal. 


\section{Experiment 6}

Monkey 3. After the animal had recovered its normal motor ability on the right side following the destruction of the left cortical motor area, the right cerebral motor area was cauterized (July 28, 1916). This produced a left hemiplegic condition similar to those in the other experiments:

Management and results. Both arms and legs were permitted to be free to move, but the left paralyzed arm and leg were carefully massaged without, however, giving individual attention to the special muscle groups and to the nerves as had been done in the second part of the preceding experiment. This animal continued to use the right arm almost to the exclusion of the left, although both may now be used when it is necessary, the left more awkwardly than the right. The animal moves well, climbs and jumps, it has been seen to pick over its cage companion for parasites (?), but all of its movements are more awkward than those of monkey 2 which is in the same cage with it. There has been a slight deterioration of the right side in that the right hand cannot be used as well as at the end of the special training period, and at the present writing it exhibits a slight wrist drop on the right side, but a marked wrist drop on the left. When it handles food, which it usually takes in both hands at one time, it is noticeable that there is considerable weakness on the left, there is also a marked awkwardness. During December, 1916, this animal was noticed to have convulsions. One began December 18, 1916, at about 2.00 p.m., and the animal was under observation during the convulsive attack. The animal had been feeding, and was holding two bananas in his hands. The food was suddenly dropped, and the monkey tried to get it from the floor, but not being apparently able to do this with the hands, he lowered his head to where the bananas had fallen. In that position a series of clonic movements began. The animal fell to its side, and the convulsive movements were noticed to be especially (or entirely, it could not be said with certainty) of the right side, but the face area was not apparently involved. The leg was more active than the arm, although the arm shook all over and the fingers were also in alternate contraction and relaxation. When the convulsion had partly subsided the animal tried to crawl over a partition (about $35 \mathrm{~cm}$. high) which separated the cage into two parts. This attempt was unsuccessful at first, but the monkey continued to try until it succeeded. Success was finally attained only with great effort of the left arm and leg, and the right 
corresponding segments were dragged over. The animal returned to its food about a half minute after it had successfully negotiated the partition. For about four or five minutes subsequently the right side could be used only with great awkwardness, but at the end of that time there was an apparent return to its former ability. There was a facial cyanosis for fully half an hour subsequent to the convulsion. Another similar convulsion occurred the same evening, about 8 hours after the first one. Others have been noted repeatedly both by one of us and by an assistant, and they have appeared to be of the same character as that described. The monkey has not been able to use the right hand as well as he did previous to the occurrence of the convulsions, and some of the apparent deterioration in the proper use of that hand may reasonably be ascribed to the unknown convulsive-producing condition.4

Summary. In this experiment although general massage was given to the paralyzed segments there was less recovery than in those cases in which special attention was paid to the individual muscles and nerves. The recovery has been sufficient to enable the animal to feed himself, and to perform other necessary acts, but not sufficient to make the finer kinds of movements; the muscles remain weak. This case is complicated with a unilateral epileptiform condition, which may have been the reason for a slight deterioration in the use of the right hand.

4 Since the above was written another convulsion bas occurred in the presence of one of us, and its characters have been noted. The monkey had been eating a piece of carrot for about three minutes, when the food which he had been holding was dropped to the floor, the right hand was clenched to make a fist, then there was a tonic flexion of the forearm on the arm, and this was followed by a slow tonic abduction of the arm to about 75 degrees from the normal position. A sudden relaxation then occurred, followed by a series of clonic movements in the whole of the arm area, and at this time the monkey cried several times. From this time the convulsion was purely clonic, the right leg following the arm, and in a few seconds the left side followed the right in a series of severe and extensive movements. The convulsion ended in 27 or 28 seconds, with a gradual lessening of the rate and of the extension of the movements, and as soon as the clonic movements had stopped the animal took up the piece of carrot which it had been eating previous to the attack. The convulsions have been coming at longer intervals, and they have been observed chiefly after the animal begins his morning meal. 


\section{Experiment 7}

Monkey 4. On July 25, 1916, the left motor cortex was destroyed in the manner previously described. This brought about a right-sided hemiplegia, with flaccidity of the arm and leg.

Management and results. The movements of the left arm were prevented by tightly bandaging that arm to the trunk. The special treatment consisted in the stimulation of the extensors of the arm, the shoulder muscles, and the muscles of the leg by friction, by the stimulating of the corresponding nerves, and by the irritating exercises to

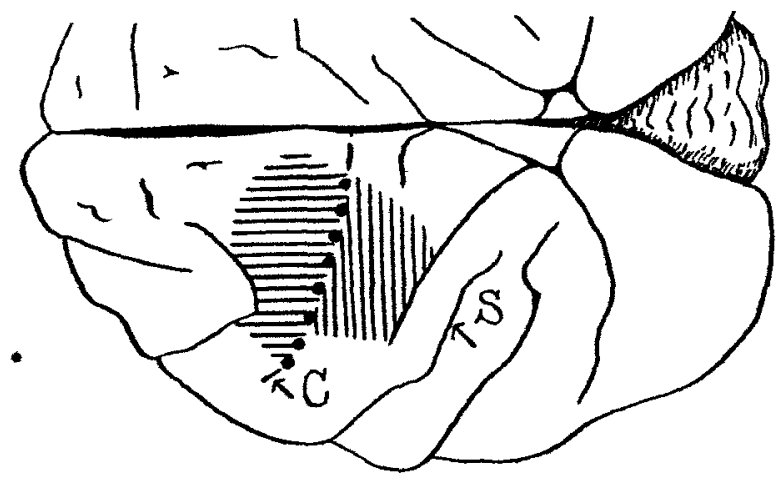

Fig. 1. Part of the Superior Surface of the Brain of Monkey 4

The obviously destroyed motor area is indicated by horizontal lines, and the apparently abnormal post-central area indicated by vertical lines. $C$, central fissure, partly indeterminate and indicated by the dotted line; $\mathrm{S}$, fissure of Sylvius. About natural size.

cause the animal to make defensive and offensive movements. The recovery was rapid, after the first few days the improvement being marked. After three weeks of the treatment (August 18) it was not possible to notice any difference in the activities of the two sides of the body, the right arm being used as well as the left for such operations as feeding and climbing, and the right leg being perfectly controlled and coordinated in walking, running, climbing, and jumping.

This animal had been sent from Washington to New Hampshire, and on August 20 it was noticed to have coryza. This developed like an influenza with pulmonary symptoms, and the animal died three days later. The brain was removed and the accompanying diagram illus- 
trates the condition of the left hemisphere. The microscopic examination has not been made at this writing, but the gross appearance shows that most, if not all, of the arm and leg areas of the left cortex had been destroyed. The precental area which showed destruction is shown in the diagram by horizontal lines. Behind the central fissure there is another area (post-central and intermediate post-central, according to Campbell's histological differentiation) which appears to be affected. This area may have been involved because of changes in the blood supply in the application of the cautery to the precentral cortex, although it is not possible to determine the matter until the brain has been examined histologically. It also appears from the gross examination that some of the uppermost part of the leg area may have escaped destruction because of its proximity to the longitudinal sulcus, but this also cannot be definitely determined until the results of the microscopical examination are available. It has previously been noted that the cautery was pushed into the brain so as to destroy the parts not usually accessible, and it may be that the superficial normal appearance will not be borne out by further examination.

Summary. Monkey 4 was made hemiplegic on the right side, and after three weeks, treatment of the arm and leg muscles, and by compelling the animal to use the right side, it became able to use the right arm and leg as well as a normal animal. The brain showed extensive destruction of the precentral area on the left, with a possible complication of the post-central area on the same side.

\section{GENERAL SUMMARY AND DISCUSSION}

The seven cases of hemiplegia in the four animals were treated in different ways in order to determine some of the conditions favorable to the recovery of voluntary motor function. The second experiment shows that motor recovery after the production of an hemiplegia does not result if the animal is left to its own devices, and this management (or lack of management) it is almost unnecessary to remark is what is given to most human paralytic cases. Even though the animal be prevented from using the sound (unparalyzed) segments there is little difference in the improvement from that in which no treatment is given unless in addition to the limitation of the possibility of movement there be added some extra stimulation to the muscles 
and nerves of the paralyzed side (experiment 5), although the recovery is rapid in such a case if treatment by muscle stimulation and nerve vibration be directed to the involved parts and if special stimulation exercises be given to the animal which will provoke the animal to move the paralyzed segments (final part of experiment 5). The method of treatment recommended by neurologists, general massage, does produce a slight amount of improvement but not to an extent to enable the animal to use the arm and hand properly for such ordinary operations as feeding and climbing, although these activities may be carried out after such treatment in an awkward manner. When, however, efforts are directed to the special nerves and muscles, and when the sound side of the animal is restrained so that movements of chimbing and feeding must be made, if at all, by the use of the paralyzed segments the improvement is rapid and the recovery is practically complete (experiments 1,4 , and 7 ).

One fact that stands out prominently is that recovery from the hemiplegic state may be very rapid. It has long been known that an hemiplegic monkey left to its own devices will after a considerable period of time recover the ability to use the arm and leg, but this period is one of months and is well illustrated in the one of the experiments described (experiment 2), where the animal after six months has not recovered to any great extent the ability to use the paralyzed left side. The rapid recovery of the animals used in experiments 1,4 , and 7 , and in the last part of experiment 5 , is suggestive, and perhaps conclusion-compelling, that the continued paralysis of animals, and by analogy the persistence of motor incapacities in man, is due to lack of management rather than to a real inability."

The results also suggest a reconsideration of the whole problem of cerebral motor control, and especially that of cortical motor control. It has long been believed and taught that the cerebral cortex is necessary for the production of a voluntary

\footnotetext{
- Each of the authors has in preparation a report of a series of cases of paralysis in man which will be published shortly, both showing that considerable improvement may result from properly directing the attack against certain muscle groups and their related nerves.
} 
movement. While it would be too venturesome to say from the experiments on the monkeys that the power of purely "voluntary" movement was recovered, the experiments on man which have previously been cited, and those which will later be published, show conclusively that such "voluntary" movements may be produced even though the paralysis has been what neurologists call "residual," and in some cases even when it has persisted for a decade or more. It is, however, reasonable to suppose that not all of the "recovered" motor ability of the monkeys is of the nature of reflexes of a complicated type, and if we conclude that only a few of the recovered movements are "voluntary" it is sufficient to cause us to hesitate to accept the generally accepted view of cortical motor function.

Here also may be cited the results which have been reported by von Monakow regarding the pyramidal fibers, for he finds that after the complete destruction of the motor cortex there is approximately from 25 to 33 per cent of the pyramidal fibers intact, or rather undegenerated. This fact would point, assuming the pyramidal fibers to be purely motor, to the conclusion that other parts of the brain normally send impulses to the anterior horn cells, and that the control of the body musculature is not entirely from the so-called precentral region, and it may be not entirely cortical. There remains from this anatomical argument the question of "voluntary" and "involuntary" movements, but this is more completely answered by the results of the present series of experiments as well as by the results of the experiments with human paralytics to which reference has been made.

The results are of interest in another direction, in that they place in the hands of the experimenter the means for the rapid recovery of motor function so that the "vicarious" functions of other cerebral parts may be investigated. If there is a delay in the recovery for periods of six to twelve months the possibilities of experimentation are greatly reduced. With the possibility of producing such rapid recoveries as we have described in this paper there is opened up the means of investigating certain motor functions which were not feasible previously 
on account of the long delays, in which there are numerous chances of intercurrent affections taking off some of the animals. Some of these problems have been planned and it is hoped that results will be obtained for a future article using at least two of the animals which have been reported upon here. It is because of this that the brains of the animals now alive have not been taken out and illustrated, but it is expected that a full report upon them will be made at a subsequent time.

The illustrations which are reproduced are selections of photographs of the animals at different stages. Some of the original photographs were small, and they were enlarged. To make the illustrations stand out well, the backgrounds on some of the negatives were "blocked out" and prints made from them in that condition. In other cases after prints had been made the figures of the animals were cut out. From the photographs without backgrounds the illustrations have been made. Although some of the fine detail at the edges of the prints are lost, the principal characteristics have been retained unchanged for there was"no retouching of the negatives beyond the changes in the background. 


\section{PLATE 1}

Fig. A. Monkey 1, immediately after the second operation, after the recovery of the right side, showing the paralysis on the left. Note the manner in which the left hand is turned under and the left leg outspread.

Fig. B. Monkey 1, after the second operation. The left arm is now being used as a prop as the animal sits upright.

Figs. C. and D. Monkey 1, after second operation, showing the ability of the animal to use the right (recovered) hand for holding a strap which it is trying to chew. In botb illustrations the animal is shown after the strap had been pulled upon so that the animal was irritated. Note the apparent helplessness of the left arm and leg, both of which members are flaccid.

Fig. E. Monkey 2, after the second operation. The left arm of the animal, which has been just paralyzed, was accidentally caught in the strap which was pulled upon to get the animal to sit upright. The right arm is bandaged to the body. Note the utter helplessness, there being no effort to get the left arm out from the restraining strap.

Fig. F. Monkey 2, after the second operation. Note that the animal has now recovered the ability to use the left hand and arm to a certain extent, since it holds the strap.

Fig. G. Monkey 2, after the second operation. Note that the animal now uses its left hand and arm with apparent ease to support itself in walking.

Fig. H. Monkey 2, 26 days after the second operation. The animal was photographed in the act of attempting to take hold of the irritating strap. Note that the left arm is now used in a normal manner.

Fig. J. Monkey 3 , after the second operation. Note that the right side has recovered, but that the newly paralyzed left is badly used. The left arm shows wrist drop, and the small toe on the left foot is turned under.

Fig. K. Monkey 4, after first operation, 26 days. Note that the right arm is used well in holding to the strap and that the right leg takes a normal position when the animal is sitting. 
CEREBRAL MOTOR CONTROL

PLATE 1

ROBERT OGDEN AND SHEPHERD WORY FRAN?
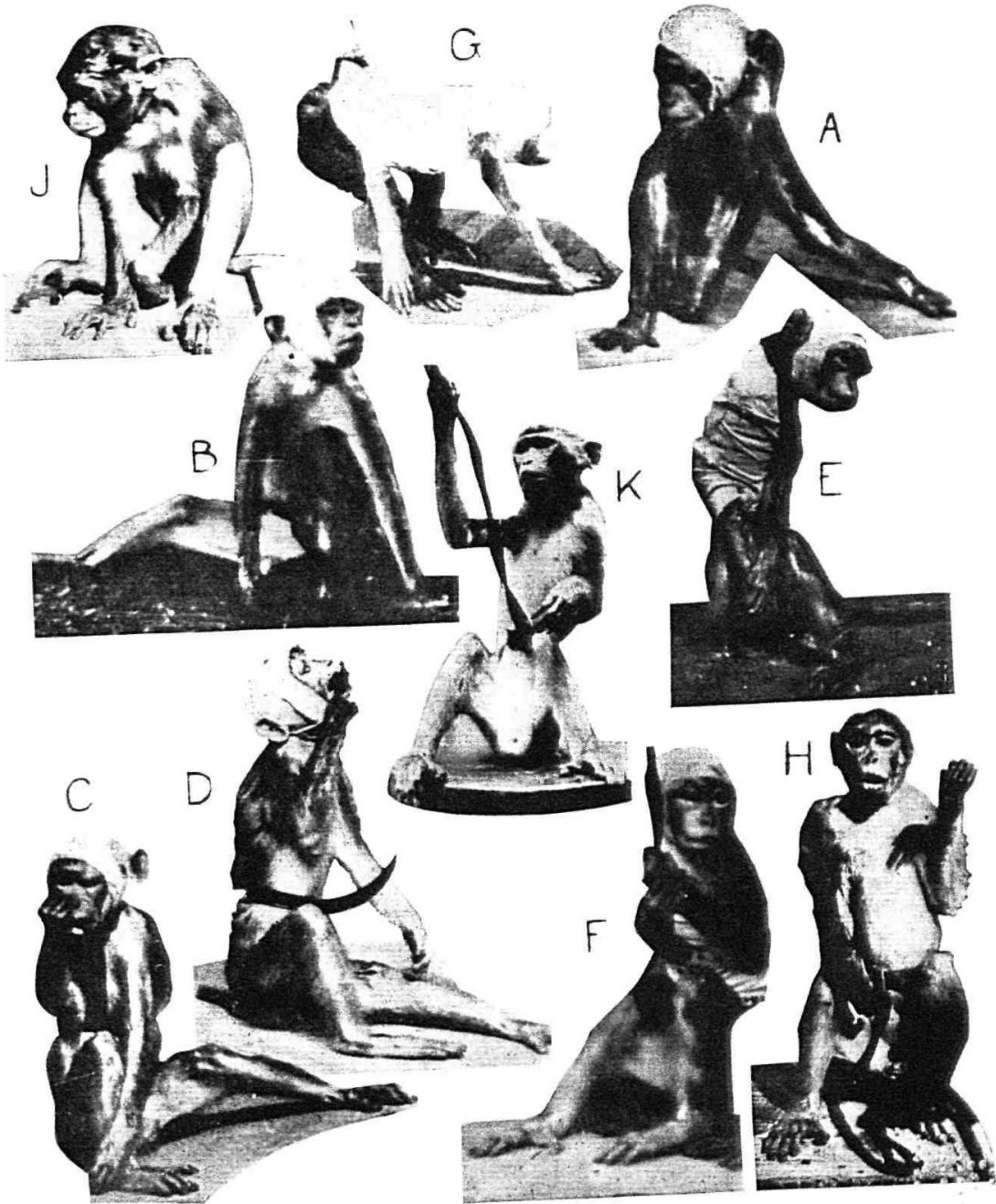